\title{
Initiation and propagation of fracture in the models of Griffith and Barenblatt
}

\author{
J.-J. Marigo ${ }^{1}$, L. Truskinovsky ${ }^{2}$ \\ 1 LPMTM, UPR-CNRS 9001, Université Paris-Nord, 93430 Villetaneuse, France \\ 2 LMS, UMR-CNRS 7649, École Polytechnique, 91128 Palaiseau, France
}

\begin{abstract}
In the setting of the simplest debonding problem we give a systematic comparison of the fracture models due to Griffith and Barenblatt. We prove that the Griffith model represents an asymptotic $\Gamma$-limit of the Barenblatt model, when the ratio of the external and internal lengths increases indefinitely. We then illustrate the character of convergence by solving explicitly two sample problems with "initially rigid" and "initially elastic" cohesive energies. The geometrical simplicity of the setting allows us to study the small parameter dependence of both global and local minimizers of the total energy.
\end{abstract}

Key words: fracture mechanics, variational methods, gamma-convergence, pull-out test, Barenblatt model

PACS: $62.20 . \mathrm{Mk}, 68.35 . \mathrm{Md}, 81.40 . \mathrm{Jj}$, 83.50.Lh

\section{Introduction}

While the well-known theories of fracture due to Griffith [1] and Barenblatt [2] take into account cohesive forces in different ways, both have the same basic structure. The theory of Griffith assumes that the surface energy density is equal to a constant that does not depend on the crack opening, while the theory of Barenblatt postulates that the surface energy density is a monotonic function of the displacement discontinuity, approaching a constant only at some sufficiently large crack opening. For consistency of the two approaches, the constant appearing in the Griffith model and representing the toughness of the material must be equal to the limiting cohesive energy in the Barenblatt model. When the two models are consistent and when Barenblatt's cohesive forces act over sufficiently short range, a formal asymptotic analysis of [3] showed that the stress fields near the tip of the crack predicted by the two models are asymptotically equivalent. In the present paper we explore the detailed character of this asymptotic convergence in the most elementary shear-lag setting of the fiber pull-off problem. The simplicity of the problem opens the way to studying the limiting behavior of both global and local minimizers without any a priori specification of the fracture geometry. An equivalent mathematical treatment of the general fracture problem presents a considerable challenge because of the complex technical issues associated with the variation of the generic discontinuity sets.

It is well known that the experimental characterization of cohesion parameters is usually based on either fiber pulling or pushing tests making the pull-out problem an important point of reference in the analysis of

Correspondence to: J.-J. Marigo (marigo@1pmtm.univ-paris13.fr) 
fiber-matrix interactions $[4,5]$. The shear-lag approximation and its dynamic extensions were derived from the three-dimensional theory in [4,6-13]. The main conclusion of these and other investigations is that the shear-lag model can be used for sufficiently long and thin fibers and that the solutions are accurate except in the vicinity of the crack tip. Although the shear-lag approximation may misrepresent some fine features of the elastic fields, the error is known to decrease with the slenderness parameter in a controlled fashion. In the present paper we compare the Griffith and Barenblatt theories by explicitly solving the shear-lag problem for a composite bar in a hard device.

The main focus of our analysis is the influence of the structure of the cohesive energy on the geometry of the discontinuity set. The deficiencies of the simplest Griffith model in predicting the crack geometry are well known. For instance, since the ultimate strength of the material in this theory is infinite, it fails to describe the infinitesimal fracture (see e.g. [14]). The problem disappears in the Barenblatt formulation, in which the ultimate strength is finite (see e.g. [15]); to achieve the same result in the Griffith theory, one needs to introduce an incremental energy minimization (see e.g. $[12,16])$. It remains unclear, however, how close are the predictions of the two theories concerning the maximally attained stress and the fine structure of the resulting fracture surface. Of particular interest is the exact domain of applicability of the much simpler and therefore more attractive Griffith approximation.

We show rigorously that the Griffith model furnishes the exact variational limit for a generic Barenblatt model when either the length of the bar tends to infinity or the internal length scale, originating from the cohesive law, tends to zero. We then predict the onset of debonding and compute the complete history of damage evolution until the full rupture. In addition to confirming asymptotic results by [3], we obtain a detailed account of the character of convergence for both the energy and the geometry of the crack. Thus, we show that the "good" quantities that are continuous in the limit include the energy and the critical stress, but not the geometrical parameters of the damage zone such as, for instance, the length of the debonding. The Barenblatt theory is shown to play a major role when the crack is about to appear; in the corresponding range of loadings the convergence of the two theories is not uniform.

Contrary to most other investigations of fracture focused on the global minimum of the energy only, we explicitly study the local minimizers. The only other comparison of the local minimizers in the theories of Griffith and Barenblatt has been previously performed for a bar in tension (see e.g. [15,17-19]). The analysis of the associated one-dimensional problem shows that, while Griffith's global minimizer asymptotically approximates Barenblatt's global minimizer, the two theories generate rather different predictions concerning the structure of the local minima of the energy. Our pull-out problem for the fiber in a hard device is both simpler and more complex than the problem for a bar in tension. It is more complex because the displacement field in both the matrix and the fiber must be considered. It is more simple because, as we show, the bifurcation from the uncracked state is always supercritical so that the only local minimizer is the global one. The special structure of the bifurcation diagram in the pull-out problem is the result of the unavoidable stress concentration around the boundary of the domain of application of the kinematic boundary condition which creates a permanent nucleation site.

The paper is organized as follows. In Sect. 2 we introduce the shear-lag energy functional and set the formal variational problem by defining the appropriate space of competitors. We then reduce the vector problem to the scalar one and prove that both local and global energy minimizers can have at most one crack. In Sect. 3 the problem is nondimensionalized and the set of dimensional parameters is reduced to two main nondimensional criteria. We then prove the main theorem establishing the Griffith theory as an exact $\Gamma$-limit of the Barenblatt theory when one of the two nondimensional parameters tends to zero. In Sect. 4 we explicitly solve the local and global minimization problem in the Griffith theory and reduce the corresponding solution to quadratures in the general Barenblatt theory. In Sect. 5 we illustrate the general solution of the Barenblatt problem by explicitly solving two special cases: with "initially rigid" cohesive response (Dugdale model) and with "initially elastic" cohesive response (piece-wise quadratic model with zero ultimate strength). Finally in Sect. 6 we present a sketch of the theory in the case of a soft device. The paper ends with a discussion of the parameter sensitivity and of the size effect. All technical proofs of the mathematical statements are collected in the Appendix. 


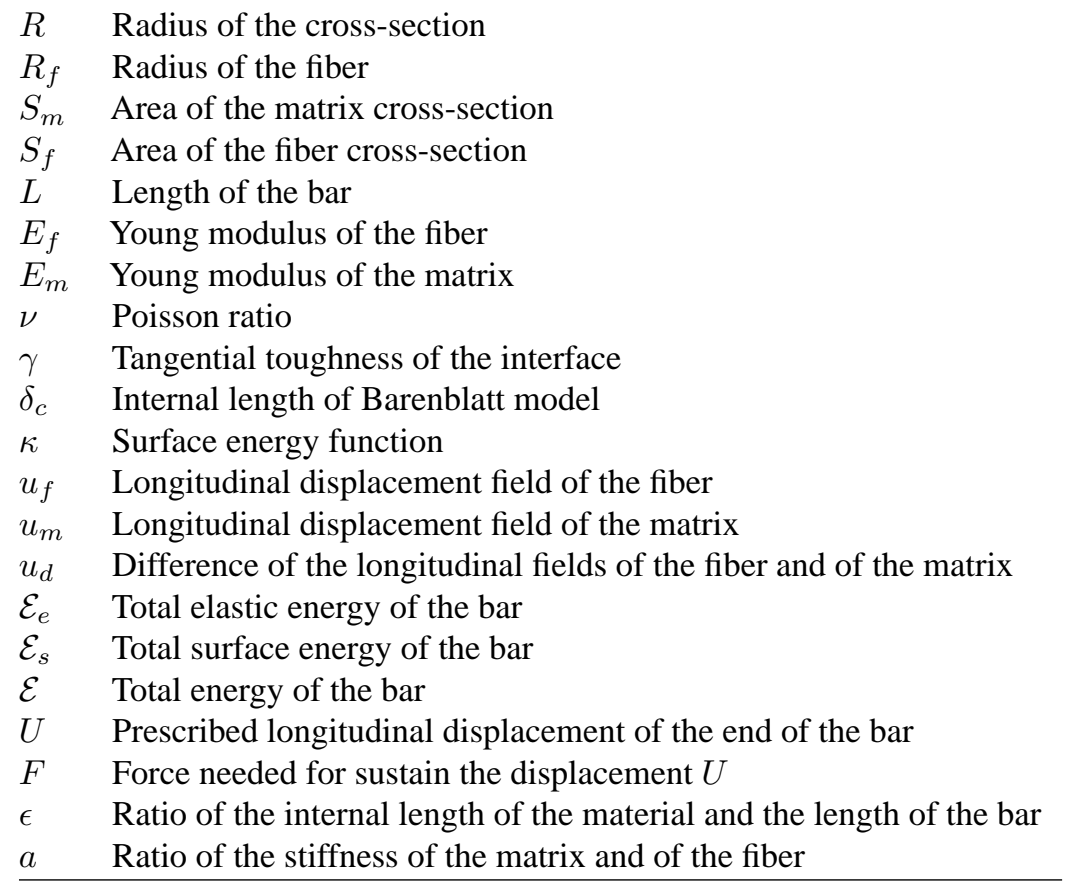

\section{Setting of the problem}

\subsection{Preliminaries}

Consider a circular cylindrical composite bar of length $L$ which includes a fiber of radius $R_{f}$ centered in the matrix of inner radius $R_{f}$ and outer radius $R$. Both the fiber and the matrix are isotropic, linearly elastic materials with Young moduli $E_{f}$ and $E_{m}$, respectively, and with the same Poisson ratio $\nu$. Both the matrix and the fiber are assumed to have infinite strength so that the fracture may only occur along the common interface. Before loading, the fiber is assumed to be glued to the matrix and the interface is assumed to be breakable. We adopt the following boundary conditions:

- the lateral part of the boundary $(r=R)$ is free;

- at one end $(z=L)$ the matrix is free, while the fiber is loaded in a hard device generating a given longitudinal displacement;

- at the other end $(z=0)$ both the matrix and the fiber are fixed.

In order to compute the equilibrium response we adopt the energy minimization point of view. Specifically, we require that, at each value of the loading parameter, the displacement field in the composite bar must deliver at least a local minimum to the corresponding energy functional. To give the precise definition of the minimization problem, we have to define

- the set of admissible displacement fields;

- the expression of the energy associated with these displacement fields;

- the norm in the set of admissible displacements allowing one to evaluate the closeness between different displacement fields.

To simplify the analysis we consider a limiting case of sufficiently slender bars and approximate the 3D problem by a 1D problem (see [6] for more details). The longitudinal displacement fields inside the fiber, $u_{f}$, and inside the matrix, $u_{m}$, are assumed to depend only on the longitudinal coordinate $z$. It is convenient to take as unknowns the pair $u=\left(u_{f}, u_{d}\right)$, where $u_{d}=u_{f}-u_{m}$. The difference of displacements $u_{d}$ vanishes if the bond remains unbroken and accordingly we can define the two sets

$$
\mathcal{D}(u)=\left\{z \in[0, L]: u_{d}(z) \neq 0\right\}, \quad \mathcal{C}(u)=\left\{z \in[0, L]: u_{d}(z)=0\right\} .
$$


The displacements $u_{f}$ and $u_{d}$ must satisfy the following kinematic boundary conditions:

$$
u_{f}(L)=U, \quad u_{f}(0)=u_{d}(0)=0,
$$

where the prescribed displacement $U$ of the end $L$ of the fiber plays the role of the loading parameter. The total energy associated with the admissible displacement fields $u=\left(u_{f}, u_{d}\right)$ contains two terms: the elastic energy $\mathcal{E}_{e}(u)$ and the cohesive (or surface) energy $\mathcal{E}_{s}(u)$. The elastic energy can be written as

$$
\mathcal{E}_{e}(u)=\frac{1}{2} \int_{0}^{L}\left(E_{f} S_{f} u_{f}^{\prime}(z)^{2}+E_{m} S_{m}\left(u_{f}^{\prime}(z)-u_{d}^{\prime}(z)\right)^{2}\right) d z,
$$

where $S_{m}=\pi\left(R^{2}-R_{f}^{2}\right)$ and $S_{f}=\pi R_{f}^{2}$ represent the area of the matrix and fiber cross-sections. For the cohesive energy we assume

$$
\mathcal{E}_{s}(u)=\int_{0}^{L} 2 \pi R_{f} \kappa\left(\left|u_{d}(z)\right|\right) d z
$$

where the presence of the absolute value means that the energy cost of sliding is independent of its sign.

We consider the following two main choices for $\kappa$ :

- Griffith model: $\kappa$ is discontinuous at zero and is constant elsewhere

$$
\kappa(\delta)=\left\{\begin{array}{l}
0, \delta=0 \\
\gamma, \delta \neq 0
\end{array}\right.
$$

- Barenblatt model: $\kappa$ is a continuous function of $\delta$ such that

$$
\kappa(0)=0, \quad \kappa(\delta)>0 \text { when } \delta>0, \quad \kappa(\infty)=\gamma .
$$

In both cases $\gamma$ represents the (tangential) toughness of the interface. For the Barenblatt model $\sigma_{c}=\kappa^{\prime}(0)$ represents the ultimate shear stress, which may either be finite ("initially rigid" cohesive response) or zero ("initially elastic" cohesive response); in the Griffith model the ultimate strength is equal to infinity. We remark that the model with finite ultimate strength appears as the natural limit of a lattice model with Lennard-Jonestype interactions (see e.g. [20]), while the model with zero ultimate strength can be linked to the presence of pre-existing fracture surfaces (see e.g. [21]).

Now notice that the total energy of the bar,

$$
\mathcal{E}(u)=\mathcal{E}_{e}(u)+\mathcal{E}_{s}(u),
$$

is finite provided that $u_{f}$ and $u_{d}$ belong to the space $W^{1,2}(0, L)$; the set of admissible displacements then consists of pairs of functions belonging to this functional space and satisfying the boundary conditions (2). This set will be denoted by $\mathcal{U}$. The precise statement of the local minimization problem reads

$$
\text { find } u \in \mathcal{U} \text { such that } \exists h>0, \forall v:\|v-u\| \leq h, \quad \mathcal{E}(u) \leq \mathcal{E}(v) \text {. }
$$

To complete the setting of the mathematical problem we choose the norm of $W^{1,2}(0, L)$ as defining the notion of a (strong) local minimum and denote it by $\|\cdot\|$. We emphasize that this assumption is of an entirely physical nature. Weaker norms, considered for instance in [15], would mean a broader set of local minimizers but would not affect the choice of the global minimum of the energy (and our main Theorem in Sect. 3.2).

\subsection{Reduction to a scalar problem}

We begin by eliminating $u_{f}$ and formulating the problem in terms of $u_{d}$ alone. Since $\mathcal{E}$ is a strictly convex function of $u_{f}$, we can minimize it out by using the following Euler-Lagrange equation:

$$
\left(E_{f} S_{f}+E_{m} S_{m}\right) u_{f}^{\prime}(z)-E_{m} S_{m} u_{d}^{\prime}(z)=\text { const }=F
$$

meaning that the normal force is constant along the entire bar. By using the boundary condition $u_{f}(0)=u_{d}(0)=$ 0 we can integrate (9) to give

$$
u_{f}(z)=\frac{A_{m}}{A} u_{d}(z)+\frac{F}{A} z
$$

Here we introduced the following notation:

$$
A_{f}=E_{f} S_{f}, \quad A_{m}=E_{m} S_{m}, \quad A=A_{f}+A_{m} .
$$


From the boundary condition $u_{f}(L)=U$, we obtain

$$
F L=A U-A_{m} u_{d}(L) .
$$

By inserting these relations in the expression of the energy, we can rewrite (7) as

$$
\hat{\mathcal{E}}\left(u_{d}\right)=\frac{A_{m} A_{f}}{2 A} \int_{0}^{L} u_{d}^{\prime}(z)^{2} d z+\frac{A}{2 L}\left(U-\frac{A_{m}}{A} u_{d}(L)\right)^{2}+2 \pi R_{f} \int_{0}^{L} \kappa\left(\left|u_{d}(z)\right|\right) d z .
$$

Here $u_{d}$ belongs to $W^{1,2}(0, L)$ and must satisfy the boundary condition $u_{d}(0)=0$. The energy $\hat{\mathcal{E}}$ is positive and hence bounded from below. Since it is also weakly lower semi-continuous, there always exists a global minimizer of the semi-linear minimization problem for (13) and therefore of the original problem (8).

\subsection{Monotonicity of the minimizers}

Proposition 1. Assume that the surface energy function $\kappa$ is an increasing function. Then the local minimizer of the energy (13) $u_{d}$ is a monotonic function of $z$, increasing when $U \geq 0$, and decreasing when $U \leq 0$.

Proposition 2. Let $u$ be a local minimizer of the energy and $\mathcal{C}(u)$ be the set of points where the fiber and the matrix are bonded. If the surface energy $\kappa$ is an increasing function, then $\mathcal{C}(u)$ is a closed interval of the form $[0, \ell]$, with $0 \leq \ell \leq L$.

Proof. The proof of Proposition 1 is given in the Appendix, whereas Proposition 2 is a consequence of the monotonicity of $u_{d}$ and of the boundary condition $u_{d}(0)=0$.

\section{Relationship between the models of Griffith and Barenblatt}

\subsection{Dimensionless formulation}

For the minimization of $\hat{\mathcal{E}}$ it is convenient to introduce dimensionless quantities. We define

$$
z=L \bar{z}, \quad u_{d}(z)=\mathcal{L} \bar{u}_{d}(\bar{z}), \quad U=\frac{A_{m}}{A} \mathcal{L} \bar{U}, \quad F=A_{m} \frac{\mathcal{L}}{L} \bar{F}, \quad a=\frac{A_{m}}{A_{f}},
$$

where

$$
\mathcal{L}=\sqrt{\frac{4 \pi R_{f} \gamma A}{A_{f} A_{m}}} L=\sqrt{\frac{4 \gamma A}{R_{f} E_{f} A_{m}}} L
$$

is the main internal length scale of the Griffith model. A nondegenerate Barenblatt model contains another internal length scale $\delta_{c}=\gamma / \kappa^{\prime}(0)$, which can be used to form a dimensionless parameter

$$
\epsilon=\frac{\delta_{c}}{\mathcal{L}}
$$

In terms of the nondimensional variables the cohesive energy in the Barenblatt model can be written as

Now, if we normalize the total energy

$$
\kappa\left(u_{d}\right)=\gamma \bar{\kappa}\left(\frac{\bar{u}_{d}}{\epsilon}\right)
$$

$$
2 \hat{\mathcal{E}}\left(u_{d}\right)=\frac{A_{f} A_{m}}{A} \frac{\mathcal{L}^{2}}{L} \overline{\mathcal{E}}\left(\bar{u}_{d}\right),
$$

we obtain

$$
\overline{\mathcal{E}}\left(\bar{u}_{d}\right)=\int_{0}^{1} \bar{u}_{d}^{\prime}(\bar{z})^{2} d \bar{z}+\int_{0}^{1} \bar{\kappa}\left(\frac{\bar{u}_{d}(\bar{z})}{\epsilon}\right) d \bar{z}+a\left(\bar{u}_{d}(1)-\bar{U}\right)^{2} .
$$

The relation between the dimensionless force $\bar{F}$ and the dimensionless total displacement takes the form

$$
\bar{F}=\bar{U}-\bar{u}_{d}(1) \text {. }
$$

As we see, the general Barenblatt problem contains two dimensionless parameters, namely $a$, which represents the relative stiffness of the matrix with respect to the fiber, and $\epsilon$, which measures the ratio of internal (cohesive) and external (geometrical) length scales. 


\subsection{Limiting behavior as $\epsilon \rightarrow 0$}

Since in the Griffith theory the nondimensional parameter $\epsilon$ is identically equal to zero, it is instructive to study how the structure of the minimizers changes as $\epsilon$ tends to 0 . One of the goals is then to show that the longer the Barenblatt composite bar is, the closer its response is to that of a composite bar with the Griffith cohesive energy.

In what follows the main parameters of the Barenblatt theory will be explicitly marked by the subscript $\epsilon$, while all other unnecessary subscripts will be dropped. For instance, we assume that $\bar{u}_{\epsilon}$ delivers a local minimum to the energy functional $\overline{\mathcal{E}}_{\epsilon}$,

$$
\overline{\mathcal{E}}_{\epsilon}(v)=\int_{0}^{1} v^{\prime}(z)^{2} d z+a(v(1)-\bar{U})^{2}+\int_{0}^{1} \bar{\kappa}\left(\frac{|v(z)|}{\epsilon}\right) d z
$$

among all $v$ from $W^{1,2}(0,1)$ such that $v(0)=0$. A local minimizer in the Griffith model can then be denoted by $\bar{u}_{0}$. By using Proposition 2 we can rewrite the corresponding energy functional $\overline{\mathcal{E}}_{0}$ in the form

$$
\overline{\mathcal{E}}_{0}(v)=\int_{0}^{1} v^{\prime}(z)^{2} d z+a(v(1)-\bar{U})^{2}+1-\operatorname{length}(\{z \in[0,1]: v(z)=0\}) .
$$

The relationship between the two theories is established by the following theorem:

Theorem 1. Suppose that $a>0, \bar{U} \in \mathbb{R}, \epsilon>0$, and $\bar{\kappa}$ is a bounded positive function which satisfies $\bar{\kappa}(0)=0$ and $\lim _{\delta \rightarrow \infty} \bar{\kappa}(\delta)=1$. Then the global minimizer of $\overline{\mathcal{E}}_{\epsilon}$ converges (strongly in the sense of the $W^{1,2}(0,1)$-norm) to the global minimizer of $\overline{\mathcal{E}}_{0}$ when $\epsilon$ goes to 0 .

Proof. The proof is given in the Appendix.

We emphasize that this theorem is rather general and remains true even if $\bar{\kappa}$ is not monotonic or not continuous. The only limitation is that it ensures the convergence of the global minimizer and does not say anything about the local minimizers. As we show in the next section, in the case of a general Barenblatt surface energy, the unique local minimizer is also the global one, and therefore our Theorem ensures that the response of the Barenblatt tends to the response of the Griffith bar unconditionally.

\section{Exact solution of the minimization problem}

As a part of the proof of Proposition 1 we have shown that, when $U=0, u=(0,0)$ is the unique local minimizer of $\mathcal{E}$. Also, by symmetry, if $u$ is a local minimizer corresponding to $U$, then $-u$ is a local minimizer corresponding to $-U$. Therefore in what follows we consider only the case $U>0$.

\subsection{Griffith model}

Owing to its simplicity, the Griffith problem can be solved in closed form. Recall that for a local minimizer $\bar{u}$ the $\operatorname{set} \mathcal{C}(\bar{u})=\left\{\bar{z} \in[0,1]: \bar{u}_{d}(\bar{z})=0\right\}$ is an interval of the form $[0, \ell(\bar{u})]$ with $0 \leq \ell(\bar{u}) \leq 1$. In the subspace of admissible displacements $\bar{v}_{d}$ in $W^{1,2}(0,1)$ such that $\bar{v}_{d}(0)=0$ and $\mathcal{C}(\bar{v})=[0, \ell]$, the energy functional of the Griffith theory reduces to

$$
\overline{\mathcal{E}}\left(\ell, \bar{v}_{d}\right)=\int_{\ell}^{1} \bar{v}_{d}^{\prime}(\bar{z})^{2} d \bar{z}+(1-\ell)+a\left(\bar{U}-\bar{v}_{d}(1)\right)^{2} .
$$

Since this functional is strictly convex with respect to $\bar{v}_{d}$ for fixed $\ell$, its unique (global) minimizer is given by

$$
\bar{u}_{d}(\bar{z})=\left\{\begin{array}{ll}
0 & , 0 \leq \bar{z} \leq \ell \\
\frac{a(\bar{z}-\ell) \bar{U}}{1+a-a \ell}, & \ell \leq z \leq 1
\end{array} .\right.
$$

To determine the value of $\ell$ we insert (24) into (23) to obtain

$$
\check{\mathcal{E}}(\ell)=\overline{\mathcal{E}}\left(\ell, \bar{u}_{d}\right)=\frac{a \bar{U}^{2}}{1+a-a \ell}+1-\ell .
$$



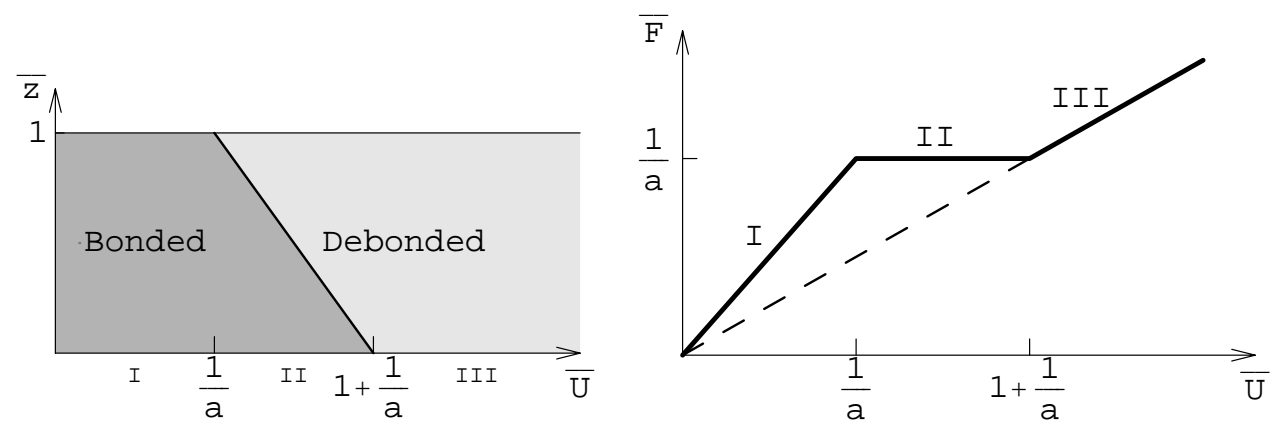

Fig. 1. Debonding process and global response in the Griffith model

Since $\check{\mathcal{E}}$ is strictly convex with respect to $\ell$, there exists one and only one local minimizer of this one-dimensional problem which is also the global one. The minimum is reached at 0 when $\check{\mathcal{E}}^{\prime}(0) \geq 0$, at 1 when $\check{\mathcal{E}}^{\prime}(1) \leq 0$, and at $\ell$ such that $\check{\mathcal{E}}^{\prime}(\ell)=0$ otherwise. Therefore, we obtain three different phases of behavior:

$$
\ell=\left\{\begin{array}{ccc}
1, & a \bar{U} & \leq 1 \quad \\
1+\frac{1}{a}-\bar{U}, 1 \leq a \bar{U} & \leq 1+a & \text { (phase II) } \\
0, & 1+a \leq a \bar{U} & \text { (phase III) }
\end{array} .\right.
$$

The global response of the composite bar can also be illustrated by the following relation between the force $\bar{F}$ and the displacement $\bar{U}$ :

$$
\bar{F}=\left\{\begin{array}{cc}
\bar{U}, \quad 0 \leq \bar{U} \leq 1 / a \quad \text { (phase I) } \\
1 / a, 1 / a \leq \bar{U} \leq 1+1 / a \text { (phase II) } \\
\frac{\bar{U}}{1+a}, \quad 1+1 / a \leq \bar{U} \quad \text { (phase III) }
\end{array}\right.
$$

which is plotted in Fig. 1. Notice that the force remains constant in the phase of progressive debonding of the fiber.

The overall behavior of the composite bar in the Griffith model can be described by the language of the theory of phase transformations. In fact, the undamaged state with energy $\overline{\mathcal{E}}_{1}(\bar{U})=\check{\mathcal{E}}(1)=a \bar{U}^{2}$ (our phase $I$ ) and the completely debonded state with energy $\overline{\mathcal{E}}_{2}(\bar{U})=\check{\mathcal{E}}(0)=1+a \bar{U}^{2} /(1+a)$ (our phase $\left.I I I\right)$ can be viewed as two different "phase modifications" of the "averaged material" of the composite bar. Now, since in the Griffith model these two "phases" can coexist without interaction, we can write for the mixed state (our phase $I I$ )

$$
\mathcal{E}\left(x, \bar{U}_{1}, \bar{U}_{2}\right)=\check{\mathcal{E}}(\ell)=x \overline{\mathcal{E}}_{1}\left(\bar{U}_{1}\right)+(1-x) \overline{\mathcal{E}}_{2}\left(\bar{U}_{2}\right),
$$

where $x=\ell / L$ is the fraction of the length of the bar occupied by the first phase. The constraint of the total displacement of the fiber can be written in the form

$$
x \bar{U}_{1}+(1-x) \bar{U}_{2}=\bar{U} .
$$

Now by minimizing the energy (28) under the constraint (29) we obtain the standard conditions of "phase equilibrium" furnishing the convexification of the total energy. The resulting overall response can be obtained through the common tangent (Maxwell) construction illustrated in Fig. 2. For a similar description of a broader class of partially damaged materials see [22].

Since in the Griffith problem the external length scale dominates, one can get an impression that in this theory the size effect is absent. This is not exactly so. Indeed, we have seen that the interface entirely debonds when the dimensionless force $\bar{F}$ reaches the value $1 / a$. In terms of the physical parameters this corresponds to a critical normal stress $\sigma_{d}$ given by (see (15))

$$
\sigma_{d}=E_{f} \sqrt{\frac{4 \pi R_{f} \gamma A}{A_{f} A_{m}}}=2 E_{f} \sqrt{1+\frac{E_{f} R_{f}^{2}}{E_{m} R_{m}^{2}}} \sqrt{\frac{\ell_{i}}{R_{f}}} .
$$

Here the parameter $\ell_{i}=\gamma / E_{f}$ with a dimension of length represents the ratio between the energy of the surface $(\gamma)$ and the energy of the bulk $(E)$. The critical debonding stress $\sigma_{d}$ is therefore proportional to the dimensionless 


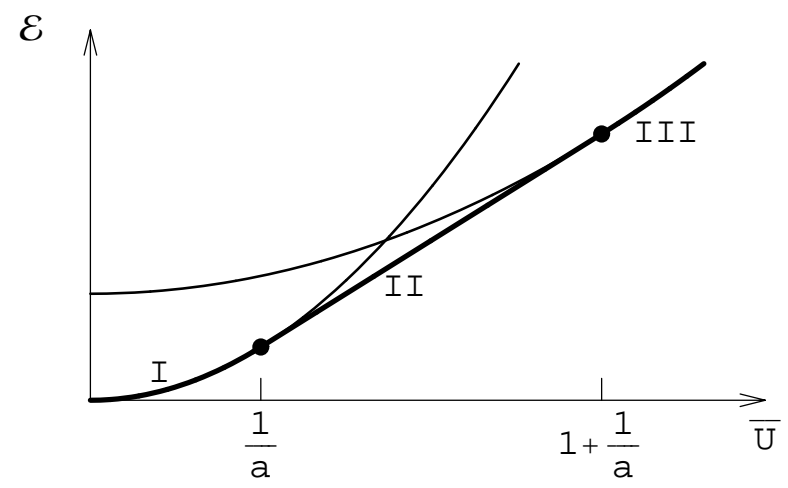

Fig. 2. The Maxwell construction providing the overall response of the composite bar in the Griffith model.

ratio $\sqrt{\ell_{i} / R_{f}}$ of the two (internal) length scales: $\ell_{i}$, associated exclusively with toughness, and $R_{f}$, giving the inner radius of the fiber. We conclude that the thicker the fiber, the lower the debonding stress.

Some important features of the debonding process will be different if instead of a hard device we consider a composite bar in a soft device. To illustrate the new possibilities we sketch in Sect. 6 the theory of a pull-out test for a Griffith fiber which is free at $z=0$ and stretched by a force $F$ at $z=L$. In particular, we find that there exists a limiting load above which no local minimizer exists. This means that the composite bar cannot support sufficiently large forces and, if overloaded, the fiber completely debonds. It is also interesting that if $a<1$, the debonding is first initiated at $z=0$ and if $a>1$, it starts at $z=1$.

\subsection{Barenblatt model}

Now assume that the surface energy is an arbitrary continuous and monotonically increasing function of $\delta$ satisfying the conditions $\kappa(0)=0, \kappa(\delta)>0$ when $\delta>0$, and $\lim _{\delta \rightarrow \infty} \kappa(\delta)=\gamma>0$. To determine the local minimizers in the general Barenblatt model we cannot use Euler-Lagrange equations because they require the surface energy function to be differentiable, eliminating for instance an important case of the Dugdale theory (see below). An alternative approach is to use a Noether identity representing a first integral of the Euler-Lagrange equations.

Proposition 3. Let $f: \mathbb{R} \rightarrow \mathbb{R}$ be a continuous function, $U \in \mathbb{R}$, and $a \geq 0$. Then a field $u \in W^{1,2}(0,1)$ such that $u(0)=0$ is a local minimum (in the sense of the norm of $W^{1,2}(0,1)$ ) of the functional I defined by

$$
I(u)=\int_{0}^{1} u^{\prime}(x)^{2}+f(u(x)) d x+a(u(1)-U)^{2}
$$

only if it satisfies the following first integral condition and boundary conditions:

$$
\begin{aligned}
& \exists C \in \mathbb{R}:-u^{\prime}(x)^{2}+f(u(x))=C, \quad \forall x \in(0,1), \\
& a U=u^{\prime}(1)+a u(1) .
\end{aligned}
$$

Proof. The proof is given in the Appendix.

By applying Proposition 3 to a general Barenblatt model, we obtain that if $\bar{u}_{d}$ is a local minimizer of $\overline{\mathcal{E}}$ it necessarily satisfies the following set of equations:

$$
\begin{aligned}
& \bar{u}_{d} \text { is increasing, } \\
& \bar{u}_{d}^{\prime}(\bar{z})^{2}-\bar{\kappa}\left(\frac{\bar{u}_{d}(\bar{z})}{\epsilon}\right)=C \quad \text { on }(0,1), \\
& \bar{u}_{d}(0)=0 \\
& \bar{u}_{d}^{\prime}(1)+a \bar{u}_{d}(1)=a \bar{U} .
\end{aligned}
$$

Due to the monotonicity of $\bar{u}_{d}$, we know that $\mathcal{C}(\bar{u})=[0, \ell]$. It is convenient to consider the cases $\ell=0$ and $\ell>0$ separately. 
- $\ell>0$. In this case, since $\bar{u}_{d}$ vanishes on $[0, \ell]$, the constant $C$ is necessarily zero. Then we obtain

$$
\bar{u}_{d}^{\prime}(\bar{z})=\left(\bar{\kappa}\left(\frac{\bar{u}_{d}(\bar{z})}{\epsilon}\right)\right)^{\frac{1}{2}} \text { on }[\ell, 1] .
$$

This differential equation can be solved explicitly once $\ell$ is known. Define $\bar{U}_{1}=\bar{u}_{d}(1)$. By using (32), (31c), and (31d), we obtain that $\ell$ and $\bar{U}_{1}$ must satisfy

$$
\begin{aligned}
& 1-\ell=g_{0}\left(\bar{U}_{1}\right) \equiv \int_{0}^{\bar{U}_{1}} \bar{\kappa}\left(\frac{u}{\epsilon}\right)^{-\frac{1}{2}} d u, \\
& \bar{U}=f_{0}\left(\bar{U}_{1}\right) \equiv \frac{1}{a}\left(\bar{\kappa}\left(\frac{\bar{U}_{1}}{\epsilon}\right)\right)^{\frac{1}{2}}+\bar{U}_{1} .
\end{aligned}
$$

Since the continuous function $f_{0}$ is strictly increasing from 0 to $\infty$ as $\bar{U}_{1}$ grows from 0 to $\infty$, (33b) has a unique solution $\bar{U}_{1}>0$ for any given $\bar{U}>0$. Concerning (33a) we must consider two possibilities depending on the behavior of the function $\bar{\kappa}(\delta)^{-\frac{1}{2}}$ near $\delta=0$ :

1. If the integral in (33a) is divergent (the case of a model with zero strength; see below), then (33a) has no solution and the minimizer must be in the other class $\ell=0$ (and $C>0$ ).

2. If the integral is convergent, the function $g_{0}$ increases continuously from 0 to $\infty$ with $\bar{U}_{1}$. Hence (33a) admits a unique solution $\ell \in(0,1]$ provided that $\bar{U}_{1} \in\left[0, \bar{U}_{1}^{c}\right)$, with $\bar{U}_{1}^{c}=g_{0}^{-1}(1)$, which in turn requires that the loading $\bar{U}$ lies in $\left[0, \bar{U}^{c}\right)$ with $\bar{U}^{c}=f_{0}\left(\bar{U}_{1}^{c}\right)$. Once $\bar{U}_{1}$ and $\ell$ are determined, the field $\bar{u}_{d}$ can be found explicitly: $\bar{u}_{d}(\bar{z})=0$ for $\bar{z} \in[0, \ell]$, and $\bar{u}_{d}(\bar{z})=g_{0}^{-1}(\bar{z}-\ell)$ for $\bar{z} \in[\ell, 1]$.

To summarize, for $0<\bar{U}<\bar{U}^{c}$, there exists a unique function $\bar{u}_{d}$ satisfying the necessary conditions of optimality (31a)-(31d) and having length $(\mathcal{C}(\bar{u}))>0$. When $\bar{U} \geq \bar{U}^{c}$, this type of a minimizer does not exist.

- $\ell=0$. Now consider local minimizers with an entire debonding of the interface. In this case, since $\bar{u}_{d}(0)=0$, we have $C=\bar{u}_{d}^{\prime}(0)^{2} \geq 0$. We consider separately the cases $C=0$ and $C>0$. We still set $\bar{U}_{1}=\bar{u}_{d}(1)$.

(i) $C=0$. We can use (33a) and (33b) with $\ell=0$ and obtain that $\bar{U}$ must satisfy $1=g_{0}\left(\bar{U}_{1}\right)$ and $\bar{U}=f_{0}\left(\bar{U}_{1}\right)$. That requires the integral of $\bar{\kappa}(\delta)^{-\frac{1}{2}}$ near $\delta=0$ to converge. Then $\bar{U}=\bar{U}^{c} \equiv f_{0} \circ g_{0}^{-1}(1)$ and the field $\bar{u}_{d}$ can be written explicitly as $\bar{u}_{d}(\bar{z})=g_{0}^{-1}(\bar{z})$ on $[0,1]$.

(ii) $C>0$. In this case we get $\bar{u}_{d}^{\prime}(\bar{z})=\left(\bar{\kappa}\left(\frac{\bar{u}_{d}(\bar{z})}{\epsilon}\right)+C\right)^{\frac{1}{2}}$ on $[0,1]$. This differential equation can be solved as soon as $C$ is known. By using (31b)-(31d), we obtain that $C$ and $\bar{U}_{1}$ must satisfy

$$
\begin{gathered}
\bar{U}=f\left(C, \bar{U}_{1}\right) \equiv \bar{U}_{1}+\frac{1}{a}\left(C+\bar{\kappa}\left(\frac{\bar{U}_{1}}{\epsilon}\right)\right)^{\frac{1}{2}}, \\
1=g\left(C, \bar{U}_{1}\right) \equiv \int_{0}^{\bar{U}_{1}}\left(\bar{\kappa}\left(\frac{u}{\epsilon}\right)+C\right)^{-\frac{1}{2}} d u .
\end{gathered}
$$

Consider the function $g\left(C, \bar{U}_{1}\right)$ for $C>0$ and $\bar{U}_{1}>0$. At fixed $C, g$ is strictly increasing with $\bar{U}_{1}$ from 0 to $\infty$, while, at fixed $\bar{U}_{1}$, it is strictly decreasing from the value $g_{0}\left(\bar{U}_{1}\right)$ - which can be infinite - to zero. Now, at fixed $C>0$, (34b) admits a unique solution $\bar{U}_{1}=G(C)$. Moreover, $G(C)$ increases from the value $g_{0}{ }^{-1}(1)-$ with the convention that $g_{0}{ }^{-1}(1)=0$ when $g_{0}$ is not finite - to $\infty$ as $C$ grows from 0 to $\infty$. Inserting the relation between $\bar{U}_{1}$ and $C$ in (34a), we obtain the following equation for $C$ :

$$
\bar{U}=f(C, G(C)) \text {. }
$$

Since, at fixed $\bar{U}_{1}, f$ increases from the value $f_{0}\left(\bar{U}_{1}\right)$ to $\infty$ as $C$ grows from 0 to $\infty$ and since, at fixed $C, f$ increases from 0 to $\infty$ as $\bar{U}_{1}$ grows from 0 to $\infty$, we conclude that the function $f(C, G(C))$ increases from the value $f(0, G(0))=f_{0}\left(g_{0}^{-1}(1)\right)=\bar{U}^{c}$ to $\infty$ as $C$ goes from 0 to $\infty$. Thus (35) admits one (and only one) solution $C>0$ for a given $\bar{U}$ provided that $\bar{U}>\bar{U}^{c}$. of

Once $\bar{U}_{1}$ and $C$ are determined, the field $\bar{u}_{d}$ can be obtained explicitly because $\bar{u}_{d}(\bar{z})$ is the unique solution

$$
\int_{0}^{\bar{u}_{d}(\bar{z})}\left(\bar{\kappa}\left(\frac{u}{\epsilon}\right)+C\right)^{-\frac{1}{2}} d u=\bar{z} .
$$



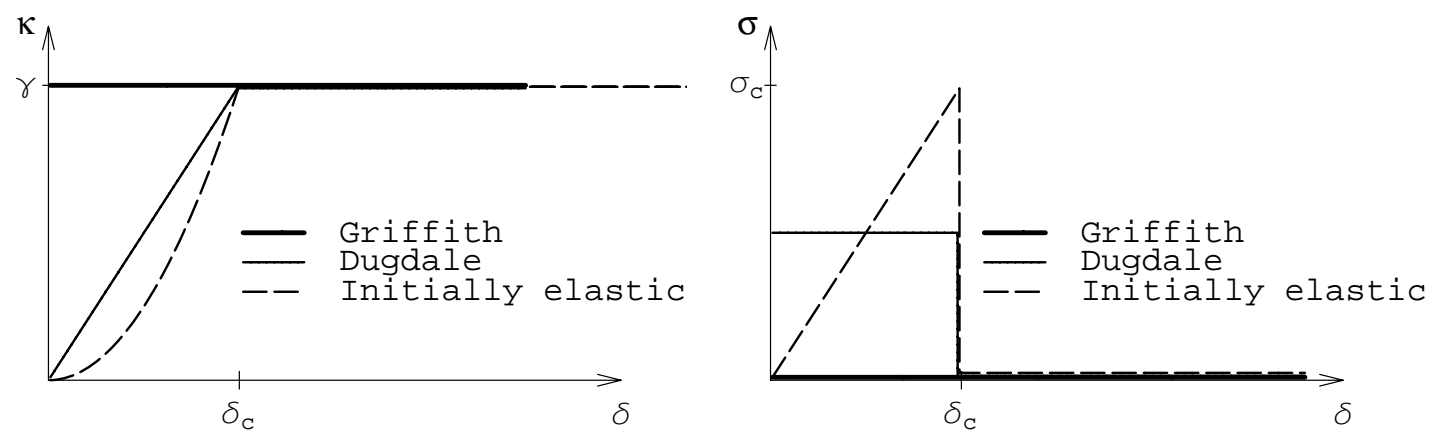

Fig. 3. Different cohesive energy functions and the corresponding cohesive stress-opening relations

This solution is obviously a strictly increasing function of $\bar{z}$. We have therefore found that for $\bar{U} \geq \bar{U}^{c}$ there exists a unique function $\bar{u}_{d}$ satisfying the necessary conditions of optimality (31a)-(31d) and having length $(\mathcal{C}(\bar{u}))=0$. Such a minimizer does not exist when $\bar{U}<\bar{U}^{c}$.

By comparing different cases, we can conclude that there exists at most one local minimizer. Since we have already shown that there is always a global minimizer, we may conclude that the only local minimizer is also the global one.

\section{Explicit examples of the minimizers in the Barenblatt problem}

To illustrate the general existence and uniqueness results for the Barenblatt model, here we present two particular examples. To cover different physical situations we consider two exactly solvable models with either "initially rigid" or "initially elastic" cohesive response (see Fig. 3). Specifically, we define

1. The Dugdale model (“initially rigid" response) :

$$
\kappa(\delta)=\left\{\begin{array}{c}
\gamma \frac{\delta}{\delta_{c}}, 0 \leq \delta \leq \delta_{c} \\
\gamma, \quad \delta \geq \delta_{c}
\end{array} .\right.
$$

2. The piece-wise linear model with zero ultimate strength ("initially elastic" response) :

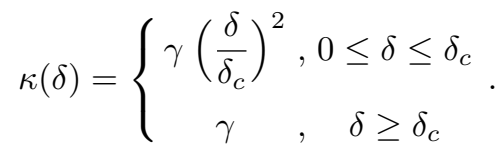

\subsection{Dugdale model}

Consider first the case where the surface energy is given by (37). The calculation of the integrals and solution of (34a) and (34b) is then straightforward. It remains to distinguish the points where the relative sliding $\bar{u}_{d}$ of the fiber is less than the critical value $\delta_{c}$ (partial debonding) from those where it is larger (total debonding). The detailed succession of various phases of debonding is different depending on the value of $\epsilon$, i.e. on whether the bar is long or short.

- Case of a long bar : $\epsilon<1 / 2$. We can distinguish three phases of debonding:

1. Phase I : $0<\bar{U} \leq \epsilon+1 / a$. The bar is totally bonded along the interval $[0, \ell]$ and partially debonded along the remaining part $(\ell, 1)$. The relation between $\ell$ and $\bar{U}$ is given by

$$
\ell=1+\frac{1}{a}-\sqrt{\frac{1}{a^{2}}+4 \epsilon \bar{U}}
$$


The relative sliding of the fiber takes the form

$$
\bar{u}_{d}(\bar{z})=\left\{\begin{array}{ll}
0 & , 0 \leq \bar{z} \leq \ell \\
\frac{(\bar{z}-\ell)^{2}}{4 \epsilon} & , \ell \leq \bar{z} \leq 1
\end{array} .\right.
$$

At the end of this phase, i.e. when $\bar{U}=\epsilon+1 / a$, the relative sliding of the fiber reaches the critical value 1 at the loaded end $\left(\bar{u}_{d}(1)=1\right)$, which marks the beginning of the second phase. During the first phase, the force $\bar{F}$ needed to sustain the displacement $\bar{U}$ is

$$
\bar{F}=\frac{2 \bar{U}}{1+\sqrt{1+4 a^{2} \epsilon \bar{U}}} .
$$

2. Phase II : $\epsilon+1 / a<\bar{U}<1-\epsilon+1 / a$. During this phase, the bar is split into three parts with perfect bonding along the interval $[0, \ell]$, partial debonding along the interval $(\ell, d)$, and total debonding along the remaining part $[d, 1]$. The two limit points are given by

$$
\ell=1-\epsilon+\frac{1}{a}-\bar{U}, \quad d=\ell+2 \epsilon .
$$

Notice that the (dimensionless) length of the partially debonded part equals $2 \epsilon$. If we return to dimensional quantities, this length is independent of the length of the bar and is equal to $\left(\frac{4 \gamma A}{R_{f} E_{f} A_{m}}\right)^{-\frac{1}{2}} \delta_{c}$. The relative displacement is now

$$
\bar{u}_{d}(\bar{z})= \begin{cases}0 & , \quad 0 \leq \bar{z} \leq \ell \\ \frac{(\bar{z}-\ell)^{2}}{4 \epsilon} & , \quad \ell \leq \bar{z} \leq d \\ \bar{z}-\ell-\epsilon & , \quad d \leq \bar{z} \leq 1\end{cases}
$$

At the end of this phase $\ell=0$, which means that the perfectly bonded domain ceases to exist, marking the beginning of the third phase. During the second phase, the force $\bar{F}$ remains constant:

$$
\bar{F}=\frac{1}{a} .
$$

3. Phase III : $\bar{U} \geq 1-\epsilon+1 / a$. Since the end $\bar{z}=0$ of the fiber is fixed, there always remains a zone in which the debonding is partial. The length of this zone decreases to 0 as $\bar{U}$ goes to infinity. Outside this partially debonded zone, the debonding is total. From an analytical point of view, it is more convenient to use $\bar{F}$ as the parameter. The location of the limit point between the two zones is given by

$$
d=2 \epsilon\left(a \bar{F}-\sqrt{a^{2} \bar{F}^{2}-1}\right),
$$

whereas the displacement field is now

$$
\bar{u}_{d}(\bar{z})=\left\{\begin{array}{ll}
\frac{(\bar{z}-\ell)^{2}}{4 \epsilon}+\bar{z} \sqrt{a^{2} \bar{F}^{2}-1} & , 0 \leq \bar{z} \leq d \\
a \bar{F} \bar{z}+d-\epsilon & , d \leq \bar{z} \leq 1
\end{array} .\right.
$$

Finally, the force $\bar{F}$ needed to sustain the displacement $\bar{U}$ is implicitly given by

$$
\bar{U}=(1+a) \bar{F}-\epsilon+2 \epsilon\left(1-a^{2} \bar{F}^{2}+a \bar{F} \sqrt{a^{2} \bar{F}^{2}-1}\right) .
$$

One can show that $\bar{F}$ strictly increases from $1 /$ a to $\infty$ as $\bar{U}$ grows from $1-\epsilon+1 / a$ to $\infty$. The explicit inversion of this relation requires solving an algebraic equation of the third order.

The three phases of the debonding process and the global response of the bar are illustrated in Fig. 4.

- Case of a short bar : $\epsilon \geq 1 / 2$. We can again distinguish three phases of debonding, the first and the last being the same as in the former case and the second corresponding to a case in which every point of the bar is partially debonded. The difference is due to the fact that the limiting point $\ell$ which separates the perfectly bonded zone from the partially debonded one reaches 0 before the totally debonded zone appears at $\bar{z}=1$. 

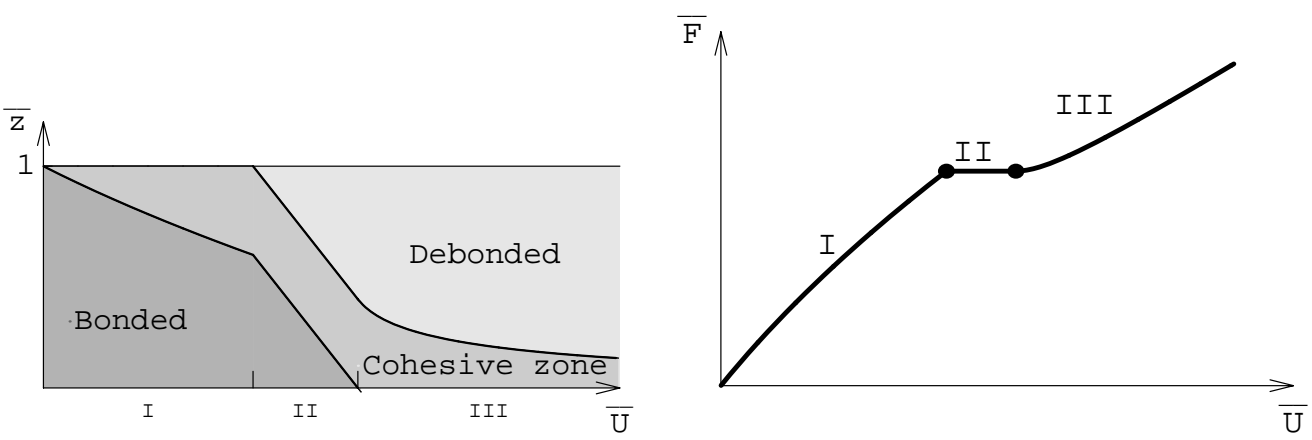

a Case of a long bar : $\epsilon<1 / 2$.
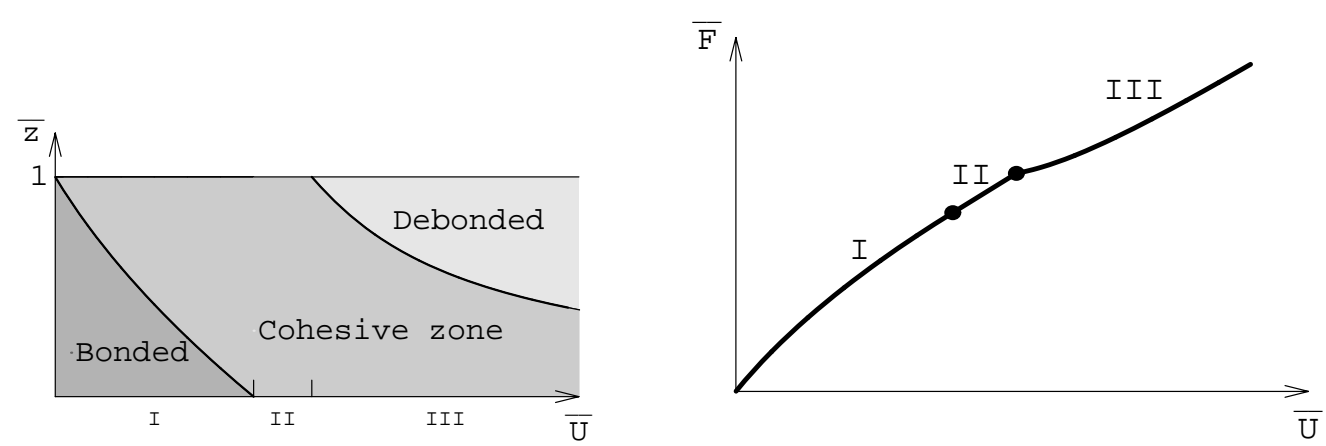

b Case of a short bar : $\epsilon>1 / 2$.

Fig. 4. Debonding process and global response in the Dugdale model

Specifically, the different phases are now delimited by

$$
\begin{cases}\text { Phase I : } \quad 0<\bar{U} \leq \frac{1}{4 \epsilon}+\frac{1}{2 a \epsilon} \\ \text { Phase II : } \quad \frac{1}{4 \epsilon}+\frac{1}{2 a \epsilon}<\bar{U} \leq \epsilon+\frac{\epsilon}{a}+\frac{1}{4 a \epsilon} . \\ \text { Phase III : } \quad \epsilon+\frac{\epsilon}{a}+\frac{1}{4 a \epsilon}<\bar{U}\end{cases}
$$

The relations giving $\ell, \bar{u}_{d}$, and $\bar{F}$ as functions of $\bar{U}$ during the first phase and those giving $d, \bar{u}_{d}$, and $\bar{U}$ as functions of $\bar{F}$ during the third phase are the same as in the case of long bars. During the second phase, the whole bar is partially debonded, and the relative displacement of the fiber is now given by

$$
\bar{u}_{d}(\bar{z})=\frac{\bar{z}^{2}}{4 \epsilon}+\frac{a}{1+a}\left(\bar{U}-\frac{1}{4 \epsilon}-\frac{1}{2 a \epsilon}\right) \bar{z}
$$

whereas the force $\bar{F}$ is no longer constant but is strictly increasing:

$$
\bar{F}=\frac{1}{1+a}\left(\bar{U}+\frac{1}{4 \epsilon}\right) .
$$

The three phases of the debonding process and the global response of a short bar are illustrated in Fig. 4.

\subsection{A piece-wise linear model with zero ultimate strength}

Finally, consider the case in which the surface energy is given by (38) and is characterized by zero ultimate stress. As we have already pointed out, in this model the integral $g_{0}$ in (33a) is divergent. As a result, the perfectly bonded phase does not exist: as soon as $\bar{U}>0$, all points of the fiber slide with respect to the matrix. As in the case of the Dugdale surface energy, we need to distinguish here the points where the relative displacement $\bar{u}_{d}$ is below the critical value $\delta_{c}$ from those where it is above. We obtain two phases of the debonding process (Phases II and III): 

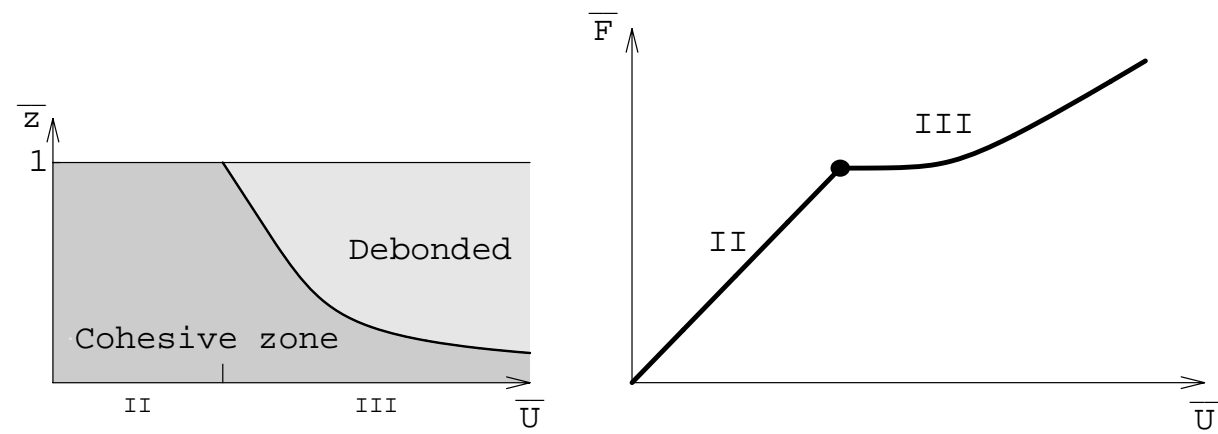

Fig. 5. Debonding process and global response in the piece-wise linear model with zero ultimate strength

1. Phase II : $0<\bar{U} \leq \epsilon+1 /(a \tanh (1 / \epsilon))$. The relative displacement of the fiber and the force $\bar{F}$ needed to sustain the total displacement $\bar{U}$ are given by

$$
\begin{gathered}
\bar{u}_{d}(\bar{z})=\frac{a \epsilon \bar{U}}{\cosh \frac{1}{\epsilon}+a \epsilon \sinh \frac{1}{\epsilon}} \sinh \frac{\bar{z}}{\epsilon}, \\
\bar{F}=\frac{\bar{U}}{1+a \epsilon \tanh \frac{1}{\epsilon}} .
\end{gathered}
$$

Both increase with $\bar{U}$. At the end of this phase, the relative displacement of the fiber at $\bar{z}=1$ reaches the critical value $\epsilon$. At this point the totally debonded zone appears and the third phase begins.

2. Phase III : $\bar{U}>\epsilon+1 /(a \tanh (1 / \epsilon))$. Since the end $\bar{z}=0$ of the fiber is fixed, there always remains a zone in which the debonding is partial. The length of this zone simply decreases to 0 as $\bar{U}$ grows to infinity. Outside this zone, the debonding is total and the fiber does not interact with the matrix. From the analytical point of view, it is again convenient to use $\bar{F}$ as the parameter. The location of the limit point between the two zones is then given by

$$
d=\epsilon \sinh ^{-1} \frac{1}{\sqrt{a^{2} \bar{F}^{2}-1}},
$$

whereas the displacement field takes the form

$$
\bar{u}_{d}(\bar{z})=\left\{\begin{array}{ll}
\epsilon \sqrt{a^{2} \bar{F}^{2}-1} \sinh \frac{\bar{z}}{\epsilon} & , 0 \leq \bar{z} \leq d \\
\epsilon+a \bar{F}\left(\bar{z}-\epsilon \sinh ^{-1} \frac{1}{\sqrt{a^{2} \bar{F}^{2}-1}}\right) & , d \leq \bar{z} \leq 1
\end{array} .\right.
$$

During this last phase, the force $\bar{F}$ needed to sustain the loading $\bar{U}$ is implicitly given by

$$
\bar{U}=\epsilon+\left(1+a-a \epsilon \sinh ^{-1} \frac{1}{\sqrt{a^{2} \bar{F}^{2}-1}}\right) \bar{F},
$$

One can show that $\bar{F}$ is a strictly increasing function of $\bar{U}$. The explicit inversion of this relation requires the solution of a transcendental equation.

The two phases of the debonding process and the global response of the bar are illustrated in Fig. 5.

\section{Soft device}

For a bar in a soft device, the general energy functional takes the form

$$
\mathcal{E}(u)=\frac{1}{2} \int_{0}^{L}\left(E_{f} S_{f} u_{f}^{\prime}(z)^{2}+E_{m} S_{m}\left(u_{f}^{\prime}(z)-u_{d}^{\prime}(z)\right)^{2}\right) d z+\int_{0}^{L} 2 \pi R_{f} \kappa\left(\left|u_{d}(z)\right|\right) d z-F u_{f}(L),
$$

where the displacement of the matrix must satisfy the boundary condition $u_{m}(0)=0$, while the displacements of the fiber are free. For each value of $F$ we are searching for the local minimizers of $\mathcal{E}$ satisfying the boundary 
conditions. By choosing the Griffith surface energy and following a procedure similar to the one described in Sect. 2, one can show that the dimensionless displacement discontinuity $\bar{u}$ must minimize the energy $\overline{\mathcal{E}}$,

$$
\overline{\mathcal{E}}(\bar{v})=\int_{0}^{1} \bar{v}^{\prime}(z)^{2} d z-2 \bar{F}(\bar{v}(0)+a \bar{v}(1))-a \bar{F}^{2}+1-\text { length }(\mathcal{C}(\bar{v})) .
$$

Notice that, contrary to the case of a hard device, the configuration with $\bar{F}=0$ is not trivial. Since $\overline{\mathcal{E}}(0)=$ $0 \leq \overline{\mathcal{E}}(\bar{v})$, with equality only at $\bar{v}=0$, the global minimum always corresponds to $\bar{u}=0$. To see that this is not the only local minimizer, consider the fields $\bar{u}=$ const $\neq 0$ corresponding to rigid translations of the fiber inside the unstretched matrix. All these fields have the same energy $\overline{\mathcal{E}}($ const $)=1$. Since in any sufficiently small neighborhood of each one of these fields a perturbed field $\bar{v}$ does not vanish, the corresponding surface energy is equal to 1 , whereas the bulk energy is non-negative. Consequently these "rigid translations" are all local minimizers and, as we prove below, there are no others. Moreover, although one can approach the trivial state by a sequence of such local minimizers, the limiting configuration $\bar{v}=0$ is separated from any of these local minimizers by an energy barrier of finite height (equal to 1 ).

Another peculiar feature of the soft device is that the energy is no longer bounded from below when $\bar{F} \neq 0$. Indeed, by taking $\bar{v}(z)=q \bar{F}$ for all $z, \overline{\mathcal{E}}(\bar{v})=-(2 q+a) \bar{F}^{2}$ and tends to $-\infty$ when $q \rightarrow \infty$ provided that $\bar{F} \neq 0$. Consequently, the global minimum does not exist as long as a nonzero force is applied. A minimizer then is necessarily a local one, if one exists at all. To find the local minimizers, we proceed as in Sects. 2 and 3.

Proposition 4. Assume for determinacy that a $<1$, meaning that the fiber is stiffer than the matrix. Then

1. If $\bar{F}=0$, the perfectly bonded state $(\bar{u}=0)$ and totally debonded states $(\bar{u}=$ const $)$ are the global and the local minimizers, respectively;

2. If $|\bar{F}|<1$, the perfectly bonded state $(\bar{u}=0)$ is the unique local minimizer;

3. If $|\bar{F}|=1$, there is a one-parametric family of local minimizers $\left\{\bar{u}^{\ell}\right\}_{0 \leq \ell<1}$,

$$
\bar{u}^{\ell}(\bar{z})=\left\{\begin{array}{ll}
\bar{F}(\ell-\bar{z}) & , 0 \leq \bar{z} \leq \ell \\
0 & , \ell \leq \bar{z} \leq 1
\end{array} .\right.
$$

This family contains the state of perfect bonding $(\ell=0)$ as well as configurations with partial debonding of the fiber along the interval $(0, \ell)$;

4. If $|\bar{F}|>1$, there are no local minimizers.

Proof. The proof is given in the Appendix.

\section{Discussion}

In this concluding section we provide some additional arguments concerning the influence of the two main parameters characterizing the cohesive law - the toughness, measured by the internal length $\ell_{i}$, and the effective strength, measured by the internal length $\delta_{c}-$ on the resulting equilibrium response of the composite bar.

The effective strength of the interface enters the description of the debonding process through the parameter $\epsilon$. When the strength decreases, the parameter $\epsilon$ also decreases. Since this is equivalent to a transition from shorter to longer bars, we can conclude that the smaller the effective strength, the closer the response of the Barenblatt bar to that of a Griffith bar. The detailed character of convergence can be illustrated in the case of the Dugdale surface energy, for which the explicit relations for the minimizer $\bar{u}_{\epsilon}$ and for the overall force $\bar{F}_{\epsilon}$ are available (see (39)-(47)). Since we are interested in the limit of small toughness, it is sufficient to consider the case $\epsilon<1 / 2$. In particular, when $0<\bar{U} \leq 1 / a$, we obtain from (39)-(41) that $\bar{u}_{\epsilon}$ converges uniformly to 0 and that $\bar{F}_{\epsilon}$ converges to $\bar{U}$, which corresponds to the first phase in the response of the Griffith bar (see (24), (26), and (27)). When $1 / a<\bar{U} \leq 1+1 / a$, we obtain from (42)-(44) that $\ell_{\epsilon}$ and $d_{\epsilon}$ converge to $\ell_{0}=1+1 / a-\bar{U}$, that $\bar{u}_{\epsilon}$ converges uniformly to 0 on $\left[0, \ell_{0}\right]$ and to $\bar{z}-\ell_{0}$ on $\left[\ell_{0}, 1\right]$, and that $\bar{F}_{\epsilon}$ remains constant and equal to $1 / a$. This corresponds to the second phase in the response of the Griffith bar. Finally, when $\bar{U}>1+1 / a$, we obtain from (45)-(47) that $d_{\epsilon}$ converges to 0 , that $\bar{u}_{\epsilon}$ converges uniformly to $a \bar{z} /(1+a)$, and that $\bar{F}_{\epsilon}$ converges to $\bar{U} /(1+a)$. This corresponds to the third phase in the response of the Griffith bar. All these phases of the pull-out test are illustrated in Fig. 6, where the overall response of the Dugdale bars is plotted for different values of the effective strength of the interface against the response of the Griffith bar with the same toughness.

The value of the slope $\bar{\kappa}^{\prime}(0)$, characterizing the ultimate strength of the surface, also has an important influence on the character of the debonding process. Indeed, 


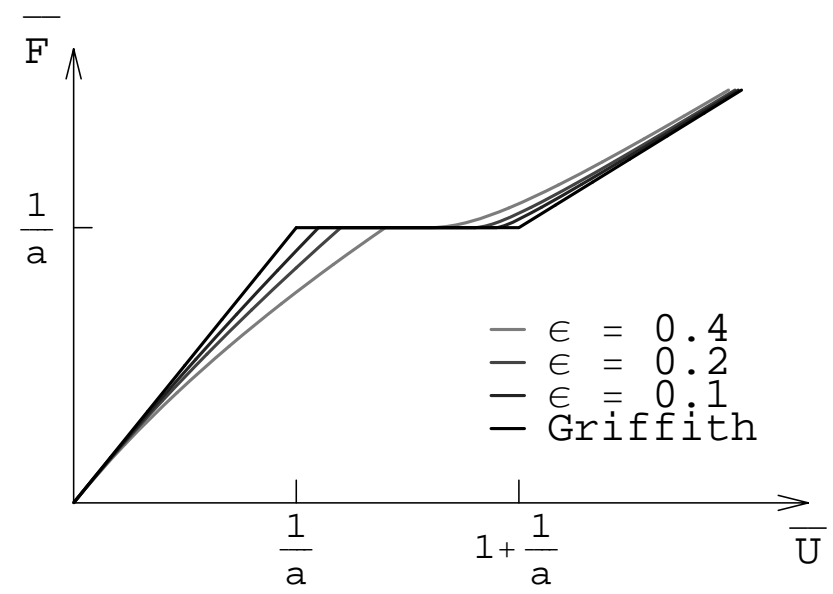

Fig. 6. Convergence of the global response of the Dugdale model to the Griffith model as the effective strength of the interface decreases

1. In the Griffith model, in which $\bar{\kappa}^{\prime}(0)=\infty$, there exists an interval of loading where the interface is entirely bonded;

2. In the model with zero ultimate strength, in which $\bar{\kappa}^{\prime}(0)=0$, a (partial) debonding appears along the entire bar as soon as the load is applied;

3. In the intermediate cases, when $0<\bar{\kappa}^{\prime}(0)<\infty$, the debonding zone appears as soon as the load is applied, but it spreads only along a finite segment of the bar. The length $1-\ell$ of this segment increases with the loading. In particular, we can compute the derivative

$$
\frac{d \ell}{d \bar{U}}(0)=\frac{2 a}{\mathcal{L} \bar{\kappa}^{\prime}(0)},
$$

confirming that the incipient debonding length is infinite when the slope $\bar{\kappa}^{\prime}(0)$ is zero ("initially elastic" model) and vanishes when the slope is infinite (Griffith model).

The above differences in the detailed character of the debonding process do not contradict our general conclusion concerning the convergence of the Barenblatt and Griffith models in the limit of zero effective strength. Thus, although in the piece-wise linear model with zero ultimate strength (but nonzero effective strength) a partial debonding appears along the entire bar as soon as the load is applied, one can show that the smaller the effective strength, the smaller the relative displacement of the fiber. In particular, in the limit of zero effective strength, the sliding disappears completely.

This discussion confirms that the important parameter determining the detailed structure of the response is the effective strength, not the ultimate strength. Notice, also, that if the pull-out test is performed for sufficiently long composite bars, then the only material parameter that can be extracted from the experiment is the (tangential) toughness of the interface which survives the transition from the Barenblatt to the Griffith description. The situation here is completely different from the one in a simple tension test, in which the structure of the set of local minimizers is much richer than in the present model and, therefore, in the absence of fluctuations (or imperfections) only the ultimate strength can be measured, the toughness playing a relatively minor role (see [19]). In that and related cases, the predictions of the Griffith model are not satisfactory and preference should be given to the Barenblatt model.

\section{Appendix}

Proof of Proposition 1. First recall that (being in $\left.W^{1,2}\right) u_{d}$ is continuous and that $u_{d}(0)=0$. Assume now that $u_{d}$ is not monotonic. Then there exist $z_{1}, z_{2}$, and $u_{0}$ such that

$$
0 \leq z_{1}<z_{2} \leq L, \quad u_{d}\left(z_{1}\right)=u_{d}\left(z_{2}\right)=u_{0}, \quad\left|u_{d}(z)\right|>\left|u_{0}\right|, \quad \forall z \in\left(z_{1}, z_{2}\right) .
$$

Let $h$ be such that $0<h<1$ and define $v_{d}^{h}$ by

$$
v_{d}^{h}(z)=\left\{\begin{array}{cr}
u_{d}(z) & , z \notin\left(z_{1}, z_{2}\right) \\
(1-h) u_{d}(z)+h u_{0}, & z \in\left(z_{1}, z_{2}\right)
\end{array} .\right.
$$


This displacement field is admissible: it satisfies the boundary condition $v_{d}^{h}(0)=0$, it is as smooth as $u_{d}$ in each subinterval delimited by $z_{1}$ and $z_{2}$, and it is continuous at $z_{1}$ and $z_{2}$, where it takes the same value $u_{0}$ as the field $u_{d}$. Let us note also that $u_{d}(L)=v_{d}^{h}(L)$ and that $\left|(1-h) u_{d}(z)+h u_{0}\right| \leq(1-h)\left|u_{d}(z)\right|+h\left|u_{0}\right|<\left|u_{d}(z)\right|$ when $z \in\left(z_{1}, z_{2}\right)$. Moreover $v_{d}^{h}$ tends to $u_{d}$ when $h$ tends to 0 . Hence, for sufficiently small $h$, it must have a larger energy than $u_{d}$. But a straightforward computation shows that

$$
\begin{aligned}
0 & \leq \hat{\mathcal{E}}\left(v_{d}^{h}\right)-\hat{\mathcal{E}}\left(u_{d}\right) \\
& =\left((1-h)^{2}-1\right) \frac{A_{m} A_{f}}{2 A} \int_{z_{1}}^{z_{2}} u_{d}^{\prime}(z)^{2} d z+2 \pi R_{f} \int_{z_{1}}^{z_{2}}\left(\kappa\left((1-h)\left|u_{d}(z)\right|+h\left|u_{0}\right|\right)-\kappa\left(\left|u_{d}(z)\right|\right)\right) d z .
\end{aligned}
$$

Since $\kappa$ is increasing, both terms on the right hand side are not positive and so they must vanish for the inequality to hold. That requires $u_{d}^{\prime}=0$ on $\left(z_{1}, z_{2}\right)$ and therefore $u_{d}(z)=u_{0}$ on $\left(z_{1}, z_{2}\right)$. This is a contradiction, so $u_{d}$ is monotonic.

It remains to be proven that $u_{d}$ increases or decreases depending on the sign of $U$. Since $u_{d}$ is monotonic and vanishes at 0 , it has the same sign on the whole interval $[0, L]$. Thus it suffices to prove that $U$ and $u_{d}(L)$ have the same sign.

Let $h>0$ and $v_{d}^{h}=(1-h) u_{d}$. Since $v_{d}^{h}$ is admissible and since $v_{d}^{h}$ converges to $u_{d}$ when $h$ goes to $0, v_{d}^{h}$ must have a larger energy than $u_{d}$ when $h$ is sufficiently small. That leads to the following inequality:

$$
\begin{aligned}
0 \leq & \left((1-h)^{2}-1\right) \frac{A_{m} A_{f}}{2 A} \int_{0}^{L} u_{d}^{\prime}(z)^{2} d z+h \frac{A_{m}}{L} U u_{d}(L)+\left((1-h)^{2}-1\right) \frac{A_{m}^{2}}{2 A L} u_{d}(L)^{2}+ \\
& +2 \pi R_{f} \int_{0}^{L}\left(\kappa\left((1-h)\left|u_{d}(z)\right|\right)-\kappa\left(\left|u_{d}(z)\right|\right)\right) d z .
\end{aligned}
$$

If $U u_{d}(L)<0$, the four terms on the right hand side are not positive and we obtain a contradiction. If $U u_{d}(L)=0$ the inequality is satisfied if and only if $u_{d}=0$. Consequently, when $U>0$, then $u_{d}(L) \geq 0$, which means that $u_{d}$ is increasing. Similarly when $U<0$, then $u_{d}(L) \leq 0$, meaning that $u_{d}$ is decreasing, and when $U=0$, then $u_{d}(L)=0$, and $u_{d}=0$ is the only possible local minimizer. In this latter case, it is easily checked that 0 is really the (unique) global minimizer.

Proof of the Theorem. The proof is divided into four steps. Essentially we are establishing a point-wise convergence of the Barenblatt functional to the Griffith functional and dealing with an elementary example of $\Gamma$-convergence (see, for instance, [23]).

(i) We first verify the basic inequality. Let $v$ be an element of $W^{1,2}(0,1)$ such that $v(0)=0$ and let $v_{\epsilon}$ be a sequence of such elements weakly converging to $v$ when $\epsilon \rightarrow 0$. Then we need to show that

$$
\overline{\mathcal{E}}_{0}(v) \leq \liminf _{\epsilon \rightarrow 0} \overline{\mathcal{E}}_{\epsilon}\left(v_{\epsilon}\right) .
$$

To prove (A1), we must consider separately the three terms in the energy expression. For the bulk elastic energy, since $0 \leq \int_{0}^{1}\left(v^{\prime}(z)-v_{\epsilon}^{\prime}(z)\right)^{2} d z$, we easily obtain that $\int_{0}^{1} v^{\prime}(z)^{2} d z \leq \liminf \inf _{\epsilon \rightarrow 0} \int_{0}^{1} v_{\epsilon}^{\prime}(z)^{2} d z$. For the boundary term, since $v_{\epsilon}(1)$ converges to $v(1)$ we get $(v(1)-\bar{U})^{2}=\lim _{\epsilon \rightarrow 0}\left(v_{\epsilon}(1)-\bar{U}\right)^{2}$. To prove the required inequality for the surface energy let $\mathcal{C}(v)$ be the set of points where $v$ vanishes and $\mathcal{D}(v)$ the set of points where it does not. Due to the positivity of $\bar{\kappa}$, we have $\liminf _{\epsilon \rightarrow 0} \int_{\mathcal{C}(v)} \bar{\kappa}(|v(z)| / \epsilon) d z \geq 0$. Furthermore, since $\lim _{\epsilon \rightarrow 0} \bar{\kappa}\left(v_{\epsilon}(z)\right)=1$ on $\mathcal{D}(v)$, we can use Fatou's Lemma to obtain

$$
1-\text { length }(\mathcal{C}(v))=\text { length }(\mathcal{D}(v))=\int_{\mathcal{D}(v)} 1 d z \leq \liminf _{\epsilon \rightarrow 0} \int_{0}^{1} \bar{\kappa}\left(\frac{\left|v_{\epsilon}(z)\right|}{\epsilon}\right) d z .
$$

This confirms that (A1) holds.

(ii) Now we need to ensure the existence of a recovery sequence. It suffices to show that, for every $v$ in $W^{1,2}(0,1)$, that

$$
\overline{\mathcal{E}}_{0}(v)=\lim _{\epsilon \rightarrow 0} \overline{\mathcal{E}}_{\epsilon}(v) .
$$

Notice first that only the surface energy depends on $\epsilon$. Since the surface energy function vanishes at 0 , we need to prove that $\lim _{\epsilon \rightarrow 0} \int_{\mathcal{D}(v)} \bar{\kappa}(|v(z)| / \epsilon) d z=$ length $(\mathcal{D}(v))$. But this follows from the assumption that $\bar{\kappa}(|v(z)| / \epsilon)$ converges to 0 if $v(z)=0$ and to 1 if $v(z) \neq 0$. Since $\bar{\kappa}$ is bounded, we can apply the Lebesgue dominated convergence theorem and the result follows. 
(iii) We can now prove that a global minimizer $\bar{u}_{\epsilon}$ of $\overline{\mathcal{E}}_{\epsilon}$ weakly converges to the global minimizer $\bar{u}_{0}$ of $\overline{\mathcal{E}}_{0}$. Since $\overline{\mathcal{E}}_{\epsilon}\left(\bar{u}_{\epsilon}\right) \leq \overline{\mathcal{E}}_{\epsilon}(0)=a \bar{U}^{2}$, the sequence $\bar{u}_{\epsilon}$ is bounded in $W^{1,2}(0,1)$ and hence we can extract a subsequence (also denoted $\bar{u}_{\epsilon}$ ) which weakly converges to a certain $\bar{u}$. Then, due to (A1), (A2), and the fact that $\bar{u}_{\epsilon}$ is a minimizer of $\overline{\mathcal{E}}_{\epsilon}$, we obtain for any $v$

$$
\overline{\mathcal{E}}_{0}(\bar{u}) \leq \liminf _{\epsilon \rightarrow 0} \overline{\mathcal{E}}_{\epsilon}\left(\bar{u}_{\epsilon}\right) \leq \lim _{\epsilon \rightarrow 0} \overline{\mathcal{E}}_{\epsilon}(v)=\overline{\mathcal{E}}_{0}(v),
$$

which ensures that $\bar{u}$ is the unique global minimizer of $\overline{\mathcal{E}}_{0}$, and therefore that $\bar{u}=\bar{u}_{0}$.

(iv) By choosing $v=\bar{u}_{0}$ in (A3) we obtain that $\overline{\mathcal{E}}_{0}\left(\bar{u}_{0}\right) \leq \lim _{\epsilon \rightarrow 0} \overline{\mathcal{E}}_{\epsilon}\left(\bar{u}_{\epsilon}\right)$ and hence that $\int_{0}^{1} \bar{u}_{\epsilon}^{\prime}(z)^{2} d z$ converges to $\int_{0}^{1} \bar{u}_{0}^{\prime}(z)^{2} d z$. That ensures that $\bar{u}_{\epsilon}$ converges strongly to $\bar{u}_{0}$.

Proof of Proposition 3. The analysis below is based on a standard use of inner variations (see [24] for a somewhat different derivation in a more general case).

The minimizers must belong to the closed subspace of $W^{1,2}(0,1)$ satisfying $u(0)=0$. Let $v$ be in $\mathcal{C}_{0}^{\infty}(0,1)$ and $h \in \mathbb{R}$. When $|h|$ is sufficiently small, $x \mapsto \phi_{h}(x)=x+h v(x)$ is a direct diffeomorphism onto [0,1]. Moreover, if $u$ is in $W^{1,2}(0,1)$ and $u(0)=0$, so is $u_{h}=u \circ \phi_{h}^{-1}$, and $u_{h}$ converges to $u$ when $h$ goes to 0 . Consequently, if $u$ is a local minimizer of $I$, the inequality $I\left(u_{h}\right) \geq I(u)$ must hold for $h$ sufficiently small. A straightforward calculation gives

$$
I\left(u \circ \phi_{h}^{-1}\right)=\int_{0}^{1}\left(\frac{u^{\prime}(x)^{2}}{\phi_{h}^{\prime}(x)}+\phi_{h}^{\prime}(x) f(u(x))\right) d x+a(u(1)-U)^{2} .
$$

By noticing that $I\left(u \circ \phi_{h}^{-1}\right)$ is now differentiable with respect to $h$ and that it has a minimum at $h=0$, we can conclude that its derivative at 0 must vanish. This leads to

$$
0=\frac{d I\left(u \circ \phi_{h}^{-1}\right)}{d h}(0)=\int_{0}^{1}\left(-u^{\prime}(x)^{2}+f(u(x))\right) v^{\prime}(x) d x, \quad \forall v \in \mathcal{C}_{0}^{\infty}(0,1) .
$$

Now it is straightforward to show that there exists a constant $C$ such that $u^{\prime}(x)^{2}-f(u(x))=C$ for all $x \in(0,1)$.

Let us derive the boundary condition. Let $u$ be a local minimizer, $h>0, \theta \in \mathbb{R}$, and define $u_{h}$ by

$$
u_{h}(x)=\left\{\begin{array}{ll}
u(x), & \text { if } 0 \leq x \leq 1-h \\
u(x)+\theta(x-1+h), & \text { if } 1-h \leq x \leq 1
\end{array} .\right.
$$

Notice that for $h$ sufficiently small, $u_{h}$ is admissible and converges to $u$ as $h$ goes to 0 ; then we can obtain the following inequality:

$$
0 \leq \int_{1-h}^{1}\left(2 \theta u^{\prime}(x)+\theta^{2}+f\left(\left|u_{h}(x)\right|\right)-f(|u(x)|)\right) d x+2 a h \theta u(1)+a h^{2} \theta^{2}-2 a h \theta U .
$$

Dividing by $h$ and passing to the limit when $h$ goes to 0 we obtain that $0 \leq 2 \theta\left(u^{\prime}(1)+a u(1)-a U\right)+\theta^{2}$. This last inequality will be satisfied for all $\theta$ if and only if $u^{\prime}(1)+a u(1)=a U$, which constitutes the desired boundary condition.

Proof of Proposition 4. The proof is divided into two steps. We first determine the short list of candidates by using necessary conditions of equilibrium and then select among them local minimizers. We adopt the following notation:

$$
\mathcal{B}=\left\{\bar{v} \in W^{1,2}(0,1): \mathcal{C}(\bar{v}) \neq \emptyset\right\}, \quad \mathcal{A}=\left\{\bar{v} \in W^{1,2}(0,1): \mathcal{C}(\bar{v})=\emptyset\right\}
$$

(i) The candidates. Suppose that $\bar{u}$ is a local minimizer of (57). We first show that $\mathcal{C}(\bar{u})$ is either empty or a closed interval of the form $\left[\ell_{1}, \ell_{2}\right]$, with $0 \leq \ell_{1} \leq \ell_{2} \leq 1$. Indeed, if $\mathcal{C}(\bar{u})$ is not empty, define $\ell_{1}=\min \{z \in$ $\mathcal{C}(\bar{u})\}$ and $\ell_{2}=\max \{z \in \mathcal{C}(\bar{u})\}, \mathcal{C}(\bar{u})$ being closed because $\bar{u}$ is continuous. Let $h>0$ and choose $\bar{v}_{h}$ to be the following admissible displacement field:

$$
\bar{v}_{h}(z)=\left\{\begin{array}{ll}
\bar{u}(z) & , z \notin\left[\ell_{1}, \ell_{2}\right] \\
(1-h) \bar{u}(z) & , z \in\left[\ell_{1}, \ell_{2}\right]
\end{array} .\right.
$$

Since $\bar{v}_{h}$ tends to $\bar{u}$ when $h$ goes to 0 , we must have $\overline{\mathcal{E}}(\bar{u}) \leq \overline{\mathcal{E}}\left(\bar{v}_{h}\right)$ for $h$ sufficiently small. Notice, however, that the surface energy is equal for both $\bar{v}_{h}$ and $\bar{u}$ because they vanish at the same points. The energies of the loading device for the two fields $\bar{u}$ and $\bar{v}_{h}$ are also the same, because the fields are necessarily equal at 0 and 1 . Now, 
while the bulk energies outside the interval $\left[\ell_{1}, \ell_{2}\right]$ are equal, the energy stored in the interval $\left[\ell_{1}, \ell_{2}\right]$ is less for the field $\bar{v}_{h}$ than for the field $\bar{u}$ except when $\bar{u}=0$ in this whole interval, which means that $\mathcal{C}(\bar{u})=\left[\ell_{1}, \ell_{2}\right]$.

Now examine the possibility that $\bar{u} \in \mathcal{A}$. Consider $\bar{v}_{h}=\bar{u}+h \phi, \phi \in W^{1,2}(0,1)$. For $h$ sufficiently small, $\bar{v}_{h} \in \mathcal{A}$ and $\overline{\mathcal{E}}(\bar{u}) \leq \overline{\mathcal{E}}\left(\bar{v}_{h}\right)$. This is possible only if the derivative of $\overline{\mathcal{E}}$ at $\bar{u}$ in the direction $\phi$ vanishes, meaning that $\int_{0}^{1} \bar{u}^{\prime}(z) \phi^{\prime}(z) d z=\bar{F}(\phi(0)+a \phi(1))$. This in turn requires that $\bar{F}=0$ and $\bar{u}^{\prime}=0$. We have therefore proved that a local minimizer describing the state of total debonding is possible only if $\bar{F}=0$ and that is necessarily a rigid translation of the fiber.

Finally, consider the possibility that $\mathcal{C}(\bar{u})=\left[\ell_{1}, \ell_{2}\right]$. For the corresponding displacement field $\bar{v}$ the energy

$$
\overline{\mathcal{E}}(\bar{v})=\int_{0}^{\ell_{1}} \bar{v}^{\prime}(z)^{2} d z+\int_{\ell_{2}}^{1} \bar{v}^{\prime}(z)^{2} d z-2 \bar{F}(\bar{v}(0)+a \bar{v}(1))-a \bar{F}^{2}+1-\ell_{2}+\ell_{1}
$$

is a convex functional of $\bar{v}$ and therefore the local minimizer

$$
\bar{u}(z)= \begin{cases}\bar{F}\left(\ell_{1}-z\right) & , 0 \leq z \leq \ell_{1} \\ 0 & , \ell_{1} \leq z \leq \ell_{2} \\ a \bar{F}\left(z-\ell_{2}\right) & , \ell_{2} \leq z \leq 1\end{cases}
$$

is necessarily the global one. Being projected on the family (A6) the energy becomes an affine function of $\ell_{1}$ and $\ell_{2}: \overline{\mathcal{E}}\left(\ell_{1}, \ell_{2}\right)=\left(1-\bar{F}^{2}\right) \ell_{1}+\left(1-a^{2} \bar{F}^{2}\right)\left(1-\ell_{2}\right)-a \bar{F}^{2}$. Since $\bar{u}$ depends continuously on $\ell_{1}$ and $\ell_{2}$, it is a local minimizer only if the pair $\left(\ell_{1}, \ell_{2}\right)$ delivers a global minimum to $\overline{\mathcal{E}}\left(\ell_{1}, \ell_{2}\right)$. Now, by recalling the assumption $a<1$, we obtain that, if $|\bar{F}|<1$, then $\ell_{1}=0, \ell_{2}=1$; if $|\bar{F}|=1$, then $\ell_{1} \in[0,1], \ell_{2}=1$; and finally, if $|\bar{F}|>1$, then $\ell_{1}=\ell_{2}=1$. In other words, we obtained that the local minimizers $\bar{u}$ with $\mathcal{C}(\bar{u}) \neq \emptyset$ must necessarily belong to the following set: $\bar{u}^{0}$ if $|\bar{F}|<1$, the family $\left\{\bar{u}^{\ell}\right\}_{0 \leq \ell \leq 1}$ if $|\bar{F}|=1$, and $\bar{u}^{1}$ if $|\bar{F}|>1$.

(ii) The local minimizers. Now we need to select among candidates the local minimizers. We have already seen that, when $\bar{F}=0$, all rigid translations of the fiber are local minimizers.

Consider now the candidates which belong to $\mathcal{A}$. Recall that when $|\bar{F}|<1 \bar{u}^{0}=0$ is the only candidate, and that when $|\bar{F}|=1$ all the candidates $\bar{u}^{\ell}$ have the same energy as $\bar{u}^{0}=0: \overline{\mathcal{E}}\left(\bar{u}^{\ell}\right)=\overline{\mathcal{E}}(0)=-a$. Let $x_{0} \in[0,1]$ and let $\mathcal{B}\left(x_{0}\right)=\left\{v \in W^{1,2}(0,1): v\left(x_{0}\right)=0\right\}$. Notice that $\bar{u}^{0} \in \mathcal{B}\left(x_{0}\right)$ and that $\overline{\mathcal{E}}$ admits a global minimizer in $\mathcal{B}\left(x_{0}\right)$ : the functional is bounded from below and lower semicontinous in this closed subspace of $W^{1,2}(0,1)$. If we call this (or one of these) minimizer $\bar{u}\left(x_{0}\right)$ we can follow the steps of the analysis from (i) and obtain that $\mathcal{C}\left(\bar{u}\left(x_{0}\right)\right)$ is necessarily of the form $\left[\ell_{1}, \ell_{2}\right]$. But we know also that $\bar{u}^{0}$ is the global minimizer of $\overline{\mathcal{E}}$ on the set $\mathcal{B}\left(x_{0}\right)$ when $|\bar{F}| \leq 1$ and therefore $\overline{\mathcal{E}}(0)=\overline{\mathcal{E}}\left(\bar{u}\left(x_{0}\right)\right)$. Since this property is independent of the choice of the point $x_{0}$, it remains true on the union of the sets $\mathcal{B}\left(x_{0}\right)$, that is on the whole $\mathcal{B}$. In other words, we can guarantee that when $|\bar{F}|<1 \overline{\mathcal{E}}(0) \leq \overline{\mathcal{E}}(\bar{v}), \forall \bar{v} \in \mathcal{B}$. Similarly, when $|\bar{F}|=1$ we obtain that $\overline{\mathcal{E}}\left(\bar{u}^{\ell}\right) \leq \overline{\mathcal{E}}(\bar{v}), \forall \bar{v} \in \mathcal{B}$.

To complete the proof we need to compare the energies of the candidates from $\mathcal{B}$ and $\mathcal{A}$. To prove that $\bar{u}^{0}=0$ is really a local minimizer when $|\bar{F}|<1$, it suffices to show that for any sequence $\bar{v}_{n} \in \mathcal{A}$ converging to 0 we have $\lim _{n \rightarrow \infty} \overline{\mathcal{E}}\left(\bar{v}_{n}\right)>\overline{\mathcal{E}}(0)$. Since for such sequences $\lim _{n \rightarrow \infty} \overline{\mathcal{E}}\left(\bar{v}_{n}\right)=\overline{\mathcal{E}}(0)+1, \bar{u}^{0}$ is a local minimizer. To prove that $\bar{u}^{\ell}$ is a local minimizer when $|\bar{F}|=1$, it suffices to show that for any sequence $\bar{v}_{n} \in \mathcal{A}$ converging to $\bar{u}^{\ell}$ we have $\lim _{n \rightarrow \infty} \overline{\mathcal{E}}\left(\bar{v}_{n}\right)>\overline{\mathcal{E}}\left(\bar{u}^{\ell}\right)$. Since for such sequences $\lim _{n \rightarrow \infty} \overline{\mathcal{E}}\left(v_{n}\right)=\overline{\mathcal{E}}\left(\bar{u}^{\ell}\right)+1-\ell, \bar{u}^{\ell}$ is a local minimizer when $0 \leq \ell<1$. Now, for $\ell=1$, consider the sequence $\bar{v}_{n}=\bar{u}^{1}+\bar{F} / n \in \mathcal{A}$ converging to $\bar{u}^{1}$. Since $\overline{\mathcal{E}}\left(\bar{v}_{n}\right)=\overline{\mathcal{E}}\left(\bar{u}^{1}\right)-2(1+a) \bar{F}^{2} / n<\overline{\mathcal{E}}\left(\bar{u}^{1}\right)$, the candidate $\bar{u}^{1}$ is never a local minimizer.

\section{References}

1. Griffith, A.: The phenomena of rupture and flow in solids. Philos. Trans. R. Soc. London CCXXI-A, 163-198 (1920)

2. Barenblatt, G.I.: The mathematical theory of equilibrium cracks in brittle fracture. Adv. Appl. Mech. 7, 55-129 (1962)

3. Willis, J.R.: A comparison of the fracture criteria of Griffith and Barenblatt. J. Mech. Phys. Solids 15, 151-162 (1967)

4. Marshall, D.B.: Analysis of fibre debonding and sliding experiments in brittle matrix composites. Acta Metall. Mater. 40(3), 427-442 (1992)

5. Charalambides, P.G., Evans, A.G.: Debonding properties of residually stressed brittle-matrix composites. J. Am. Ceram. Soc. 72, 746-753 (1989)

6. Geymonat, G., Krasucki, F., Marigo, J.-J.: Stress distribution in anisotropic elastic composite beams. In: Ciarlet, P.G., Sanchez Palencia, E. (eds.) Applications of Multiple Scalings in Mechanics, pp. 118-133. Masson Paris 1987

7. Hutchinson, J.W., Jensen, H.M.: Models of fibre debonding and pullout in brittle composites with friction. Mech. Mater. 9, $139-163$ (1990) 
8. Müller, I., Müller, W., Villaggio, P.: Static and dynamic pull out of an elastic rod from a rigid wall. In: Herrmann, K.P., Olesiak, Z.S. (eds.) Thermal Effects in Fracture of Multiphase Materials. (Lecture Notes in Engineering, 59), pp. 195-2002. Springer Berlin Heidelberg New York (1990)

9. Kerans, R., Parthasarathy, T.A.: Theoretical analysis of the fibre pullout and pushout tests. J. Am. Ceram. Soc. 74, 1585-1596 (1991)

10. Bechel, V.T., Sottos, N.R.: Application of debond length measurements to examine the mechanics of fiber pushout. J. Mech. Phys. Solids 46(9), 1675-1697 (1998)

11. Bilteryst, F., Marigo, J.-J.: Amorçage de la décohésion dans l'essai d'arrachement. C. R. Acad. Sci. Paris Série IIb 327, 977-983 (1999)

12. Bilteryst, F., Marigo, J.-J.: An energy based analysis of the pull-out problem. Eur. J. Mech., A/Solids 22, 55-69 (2003)

13. Cox, B.N., Sridhar, N., Beyerlein, I.: Dynamic effects in the pull-out mechanism during dynamic loading of a bridged crack. Acta Mater. 49, 3863-3877 (2001)

14. Francfort, G.A., Marigo, J.-J.: Revisiting brittle fracture as an energy minimization problem. J. Mech. Phys. Solids 46(8), 1319-1342 (1998)

15. Del Piero, G., Truskinovsky, L.: Macro- and micro-cracking in one-dimensional elasticity. Int. J. Solids Struct. 38, 1135-1148 (2000)

16. Bourdin, B., Francfort, G., Marigo, J.-J.: Numerical experiments in revisited brittle fracture. J. Mech. Phys. Solids 48, 797-826 (2000)

17. Del Piero, G., Truskinovsky, L.: A one-dimensional model for localized and distributed fracture. J. Phys. IV 8, 95-102 (1998).

18. Braides, A., Dal Maso, G., Garonni, A.: Variational formulation of softening phenomena in fracture mechanics: the onedimensional case. Arch. Rat. Mech. Anal. 146, 23-58 (1999)

19. Charlotte, M., Francfort, G., Marigo, J.-J., Truskinovsky, L.: Revisiting brittle fracture as an energy minimization problem: comparison of Griffith and Barenblatt surface energy models. In: Benallal, A. (ed.) Proceedings of the Symposium on "Continuous Damage and Fracture", pp. 7-12. Elsevier Paris (2000)

20. Truskinovsky, L.: Fracture as a phase transition. In: Batra, R., Beatty, M. (eds.) Contemporary research in the mechanics and mathematics of materials, pp. 322-332. CIMNE Barcelona (1996)

21. Xu, X.-P., Needleman, A. Numerical simulations of fast crack growth in brittle solids. J. Mech. Phys. Solids 42(9), 1397-1434 (1994)

22. Francfort, G.A., Marigo, J.-J.: Stable damage evolution in a brittle continuous medium. Eur. J. Mech., A/Solids 12(2), 149-189 (1993)

23. Braides, A.: Gamma-convergence for beginners. Oxford Lecture Series in Mathematics and Its Applications, 22. Oxford University Press Oxford (2002)

24. Truskinovsky, L., Zanzotto, G.: Ericksen’s bar revisited : energy wiggles. J. Mech. Phys. Solids 44(8), 1371-1408 (1996) 